International Journal of Pure and Applied Mathematics

Volume 98 No. $4 \quad 2015,477-484$

ISSN: 1311-8080 (printed version); ISSN: 1314-3395 (on-line version)

url: http://www.ijpam.eu

doi: http://dx.doi.org/10.12732/ijpam.v98i4.6

\title{
ON THE BEST APPROXIMATION FOR
}

\section{A 2-HYPERPLANE IN 2-INNER PRODUCT SPACES}

\author{
M. Abrishami-Moghaddam ${ }^{1 \S}$, T. Sistani ${ }^{2}$ \\ ${ }^{1}$ Department of Mathematics \\ Birjand Branch \\ Islamic Azad University \\ Birjand, IRAN \\ ${ }^{2}$ Department of Mathematics \\ Kerman Branch \\ Islamic Azad University \\ Kerman, IRAN
}

AMS Subject Classification: 46C50, 41A50

Key Words: 2-hyperplane, 2-functional, 2-norm attaining, z-representer, $z$-best approximation, $z$-proximinal, $z$-Chebyshev

\section{Introduction and Preliminaries}

Recently, some results on best approximation theory in linear 2-normed spaces have been obtained by Y. J. Cho, S. Elumalai, S. S. Kim, Sh. Rezapour and others (see [2], [4], [6], [11], [1], [13]). These papers are based on the research works in normed linear spaces made by I. Singer ([12]), S. S. Dragomir ([3]), and others. The concept which is closely related to 2-normed linear space is 2-inner product space that introduced by Diminnie, Gähler and White in 1973

Received: September 8, 2014

(C) 2015 Academic Publications, Ltd.

$\S$ Correspondence author url: www.acadpubl.eu 
and $1977([8,9])$, and has been developed extensively in different subjects by others $([5])$. In [1] the authors get some results on best approximation for convex cones in 2-inner product spaces. In this paper we show that the 2-norm attaining and has the $z$-representor are equivalent and they are equivalent with $z$-proximinality of a 2 -hyperplane.

Definition 1. Let $X$ be a linear space of dimension greater than 1 over filed $\mathbb{R}$ of real numbers. Suppose that $\langle., . \mid$.$\rangle is a \mathbb{R}$-value function defined on $X \times X \times X$ satisfying the following conditions:

a) $\langle x, x \mid z\rangle \geq 0$ and $\langle x, x \mid z\rangle=0$ if and only if $x$ and $z$ are linearly dependent,

b) $\langle x, x \mid z\rangle=\langle z, z \mid x\rangle$,

c) $\langle y, x \mid z\rangle=\langle x, y \mid z\rangle$,

d) $\langle\alpha x, y \mid z\rangle=\alpha\langle x, y \mid z\rangle$ for any scalar $\alpha \in \mathbb{R}$,

e) $\left\langle x+x^{\prime}, y \mid z\right\rangle=\langle x, y \mid z\rangle+\left\langle x^{\prime}, y \mid z\right\rangle$,

then $\langle., . \mid$.$\rangle is called a 2-inner product and (X,\langle., . \mid\rangle$.$) is called a 2-inner$ product space(or a 2-per-Hilbert space).

A concept which is closely related to 2-inner product space and introduced by Gähler in 1965, is 2-normed space [7].

Definition 2. Let $X$ be a linear space of dimension greater than 1 over filed $\mathbb{R}$ of real numbers. Suppose $\|.,$.$\| is a real-valued function on X \times X$ satisfying the following conditions:

a) $\|x, y\|=0$ if and only if $x$ and $y$ are linearly dependent vectors.

b) $\|x, y\|=\|y, x\|$ for all $x, y \in X$.

c) $\|\lambda x, y\|=|\lambda|\|x, y\|$ for all $\lambda \in \mathbb{R}$ and $x, y \in X$.

d) $\|x+y, z\| \leq\|x, z\|+\|y, z\|$ for all $x, y, z \in X$.

Then $\|.,$.$\| is called a 2-norm on \mathrm{X}$ and $(X,\|.,\|$.$) is called a linear 2-normed$ space.

It is easy to show that the 2 -norm $\|.,$.$\| is non-negative and \|x, y+\alpha x\|=$ $\|x, y\|$ for all $x, y \in X$ and $\alpha \in \mathbb{R}$. Every 2-normed space is a locally convex topological vector space. In fact for a fixed $b \in X, p_{b}(x)=\|x, b\| ; x \in X$ is a semi-norm on $X$ and the family $P=\left\{p_{b}: b \in X\right\}$ of seminorms generates a locally convex topology on $X$. 
Let $(X,\langle., . \mid\rangle$.$) be a 2$-inner product space, then we can define a 2 -norm on $X \times X$ by $\|x, y\|=\sqrt{\langle x, x \mid y\rangle}$.

A standard example of a 2-normed space is $\mathbb{R}^{2}$ equipped with the following 2-norm

$$
\|x, y\|_{s}=\text { the area of the triangle having vertices } 0, x \text { and } y .
$$

Let $(X,\|.,\|$.$) be a 2$-normed space and $W_{1}$ and $W_{2}$ be two linear subspaces of $X$. A 2-functional $\varphi: W_{1} \times W_{2} \rightarrow \mathbb{R}$ is called a bilinear 2-functional on $W_{1} \times W_{2}$, whenever for all $x_{1}, x_{2} \in W_{1}, y_{1}, y_{2} \in W_{2}$ and $\lambda_{1}, \lambda_{2} \in \mathbb{R}$,

i) $\varphi\left(x_{1}+x_{2}, y_{1}+y_{2}\right)=\varphi\left(x_{1}, y_{1}\right)+\varphi\left(x_{1}, y_{2}\right)+\varphi\left(x_{2}, y_{1}\right)+\varphi\left(x_{2}, y_{2}\right)$, ii) $\varphi\left(\lambda_{1} x_{1}, \lambda_{2} y_{1}\right)=\lambda_{1} \lambda_{2} \varphi\left(x_{1}, y_{1}\right)$.

A bilinear 2-functional $\varphi: W_{1} \times W_{2} \rightarrow \mathbb{R}$ is said to be bounded if there exists a non-negative real number $M$ (called a Lipschitz constant for $\varphi$ ) such that $|\varphi(x, y)| \leq M\|x, y\|$ for all $x \in W_{1}$ and $y \in W_{2}$. Also, the norm of a bilinear 2 -functional $\varphi$ is defined by

$$
\|\varphi\|=\inf \{M \geq 0: M \text { is a Lipschitz constant for } \varphi\} .
$$

It is known that

$$
\begin{aligned}
\|\varphi\| & =\sup \left\{|\varphi(x, y)|:(x, y) \in W_{1} \times W_{2},\|x, y\| \leq 1\right\} \\
& =\sup \left\{|\varphi(x, y)|:(x, y) \in W_{1} \times W_{2},\|x, y\|=1\right\} \\
& =\sup \left\{|\varphi(x, y)| /\|x, y\|:(x, y) \in W_{1} \times W_{2},\|x, y\|>0\right\} .
\end{aligned}
$$

Now we give some examples of linear 2-functionals as follows.

- Let $g$ and $h$ be two linear functional on $X$, then $\varphi(x, y)=g(x) h(y)$ is a linear 2-functional on $X \times X$.

- If $(X,\langle.,\rangle$.$) is an inner product space, then the function$

$$
\langle x, y \mid z\rangle=\left|\begin{array}{ll}
\langle x, y\rangle & \langle x, z\rangle \\
\langle y, z\rangle & \langle z, z\rangle
\end{array}\right|=\|z\|^{2}\langle x, y\rangle-\langle x, z\rangle\langle y, z\rangle
$$

for all $x, y, z \in X$ defined a 2-inner product on $X \times X \times X$, which is also generated the standard 2-norm $\|.,\|_{s}$. For fixed elements $y_{1}, y_{2}$ in $X$, define $\varphi$ on $X \times X$ by

$$
\varphi\left(x_{1}, x_{2}\right)=\left|\begin{array}{ll}
\left\langle x_{1}, y_{1}\right\rangle & \left\langle x_{1}, y_{2}\right\rangle \\
\left\langle x_{2}, y_{1}\right\rangle & \left\langle x_{2}, y_{2}\right\rangle
\end{array}\right|
$$

Then $\varphi$ is a bounded linear 2-functional on $X$ and $\|\varphi\|=\left\|y_{1}, y_{2}\right\|_{s}$. 
- Consider the 2-normed space $\left(\mathbb{R}^{2},\|., .\|_{s}\right)$ with the standard basis $\left\{e_{1}, e_{2}\right\}$. Define $\varphi$ by

$$
\varphi\left(x_{1}, x_{2}\right)=\left|\begin{array}{ll}
\alpha_{11} & \alpha_{12} \\
\alpha_{21} & \alpha_{22}
\end{array}\right|=\alpha_{11} \alpha_{22}-\alpha_{21} \alpha_{12}
$$

where $x_{i}=\sum_{j=1}^{2} \alpha_{i j} e_{j}, i=1,2$. Then $\varphi$ is a bounded linear 2-functional on $X$ and $\|\varphi\| \leq\left\|x_{1}, x_{2}\right\|_{s}$.

Let $\varphi$ be a nonzero linear 2-functional on $X \times V(z)$. Then we define the 2-hyperplane $H$ through the origin by

$$
H=\{x \in X ; \varphi(x, z)=0\} .
$$

Let $(X,\|.,\|$.$) be a linear 2-normed space. For a subspace G$ of $X$ let $[x, G]$ be the subspace of $X$ generated by $x$ and $G$, where $x \in X \backslash \bar{G}$. Then for $z \in X \backslash[x, G]$, an element $g_{0} \in G$ is called the $z$-best approximation element of $x$ by $G$ (with respect to $z$ ) if

$$
\left\|x-g_{0}, z\right\| \leq\|x-g, z\|
$$

for all $g \in G$. The set of all element of $z$-best approximation of $x$ by $G$ with respect to $z$ is denoted by $P_{G, z}(x)$, that is,

$$
P_{G, z}(x)=\left\{g_{0} \in G ;\left\|x-g_{0}, z\right\| \leq\|x-g, z\| \text { for all } g \in G\right\} .
$$

If for each $x \in X, P_{G, z}(x)$ is a nonempty set then $G$ is called a $z$-proximinal set and if $P_{G, z}(x)$ is exactly singleton then $G$ is called a $z$-Chebyshev set.

Let $(X,\|.,\|$.$) be a linear 2-normed space and G$ be an arbitrary nonempty subset of $X$ and $x \in X$. Then for every $x \in X$ and for every $z \in X \backslash[G, x]$ define

$$
d_{z}(x, G)=\inf _{g \in G}\|x-g, z\| .
$$

\section{Main Results}

In this section at first we give a formula for distance to a 2-hyperplane and then we show that the 2-norm attaining and has the $z$-representor are equivalent and they are equivalent with $z$-proximinality of a 2 -hyperplane. 
Theorem 3. Let $(X,\|.,\|$.$) be a linear 2-normed space, H$ a 2-hyperplane throughout the origin. Then

$$
d_{z}(x, G)=\frac{1}{\|\varphi\|}|\varphi(x, z)| .
$$

Proof. Fix any $x \in X$. For any $y \in H$ we have

$$
\frac{1}{\|\varphi\|}|\varphi(x, z)|=\frac{1}{\|\varphi\|}|\varphi(x-y, z)| \leq\|x-y, z\|
$$

implies that

$$
\frac{1}{\|\varphi\|}|\varphi(x, z)| \leq d_{z}(x, H) .
$$

Conversely, given any $\varepsilon$ with $0<\varepsilon<\|\varphi\|$, choose $y_{0} \in X$ with $\left\|y_{0}, z\right\|=1$ and $\varphi\left(y_{0}, z\right)>\|\varphi\|-\varepsilon$. Then $y=x-\frac{\varphi(x, z)}{\varphi\left(y_{0}, z\right)} y_{0}$ is in $H$ and

$$
\|x-y, z\|=\frac{|\varphi(x, z)|}{\left|\varphi\left(y_{0}, z\right)\right|} \leq \frac{|\varphi(x, z)|}{\|\varphi\|-\varepsilon}
$$

implies that

$$
d_{z}(x, H) \leq \frac{|\varphi(x, z)|}{\|\varphi\|} .
$$

From (1) and(2) we get our consequence.

Corollary 4. If $H=\{x \in X ; \varphi(x, z)=c\}$ where $c \in \mathbb{R}$. Then we have

$$
d_{z}(x, G)=\frac{1}{\|\varphi\|}|\varphi(x, z)-c| .
$$

Proof. Choose any $x_{0} \in H$ and define

$$
H_{0}:=H-x_{0}=\{y \in X ; \varphi(y, z)=0\} .
$$

Now by above theorem we have:

$$
\begin{aligned}
d_{z}(x, H) & =d_{z}\left(x-x_{0}, H-x_{0}\right)=d_{z}\left(x-x_{0}, H_{0}\right) \\
& =\frac{1}{\|\varphi\|}\left|\varphi\left(x-x_{0}, z\right)\right|=\frac{1}{\|\varphi\|}|\varphi(x, z)-c| .
\end{aligned}
$$

Definition 5. A linear 2-functional $\varphi$ is said to attain its 2-norm if there exists $x \in X$ with $\|x, z\|=1$ and $\varphi(x, z)=\|\varphi\|$. 
Definition 6. An element $x \in X$ is called a z-representer for a 2functional $\varphi$ if $\varphi(y, z)=\langle y, x \mid z\rangle$ for each $y \in X$.

Theorem 7. Let $\varphi$ be a linear 2-functional. Then $\varphi$ has a $z$-representer if and only if $\varphi$ attains its 2-norm.

Proof. If $\varphi=0$, then $x=0$ is the $z$-representer for $\varphi$, and $\varphi$ attains its 2 -norm at any $y \in X$ with $\|y, z\|=1$. Thus we assume $\varphi \neq 0$. By replacing $\varphi$ with $\frac{\varphi}{\|\varphi\|}$, we may assume that $\|\varphi\|=1$. Let $\varphi$ have the $z$-representer $x$. Then $\|x, z\|=1$ and $\varphi(x, z)=\langle x, x \mid z\rangle=\|x, z\|=1=\|\varphi\|$. Thus $\varphi$ attains its 2-norm at $x$.

Conversely, suppose $\varphi$ attains its 2 -norm at $x_{1}$, and let $M=\operatorname{ker} \varphi=\{x \in$ $X \mid \varphi(x, z)=0\}$. If $y_{0}=x-\frac{\varphi(x, z)}{\|\varphi\|} x_{1}$, Since

$$
\left\|x-y_{0}, z\right\|=\left\|\frac{\varphi(x, z)}{\|\varphi\|} x_{1}, z\right\|=\frac{|\varphi(x, z)|}{\|\varphi\|}\left\|x_{1}, z\right\|=\frac{|\varphi(x, z)|}{\|\varphi\|}=d_{z}(x, M)
$$

so $y_{0}=P_{M, z}(x)$ and $x_{1} \in M^{\perp_{z}}$, where $M^{\perp_{z}}=\{y \in X \mid\langle y, x \mid z\rangle=0 ; \forall x \in M\}$. Then we have

$$
\begin{aligned}
0=\left\langle y_{0}, x_{1} \mid z\right\rangle & =\left\langle x-\frac{\varphi(x, z)}{\|\varphi\|} x_{1}, x_{1} \mid z\right\rangle \\
& =\left\langle x, x_{1} \mid z\right\rangle-\frac{\varphi(x, z)}{\|\varphi\|}\left\langle x_{1}, x_{1} \mid z\right\rangle \\
& =\left\langle x, x_{1} \mid z\right\rangle-\frac{\varphi(x, z)}{\|\varphi\|}
\end{aligned}
$$

therefore

$$
\varphi(x, z)=\left\langle x, x_{1} \mid z\right\rangle
$$

and this show that $\varphi$ has a $z$-representer.

Theorem 8. Let $X$ be a 2-inner product space, $\varphi$ a non-zero linear 2functional, $c \in \mathbb{R}$ and $H=\{y \in X \mid \varphi(y, z)=c\}$. Then the following statements are equivalent.

(1) $H$ is z-Chebyshev.

(2) $H$ is z-proximinal.

(3) Some $x \in X \backslash[H, z]$ has a $z$-best approximation in $H$.

(4) $\varphi$ attains its 2-norm. 
(5) $\varphi$ has a $z$-representer in $X$.

Moreover, if $H$ is $z$-Chebyshev, then

$$
P_{H, z}(x)=x-\frac{(\langle x, y \mid z\rangle-c)}{\|y, z\|^{2}} y
$$

for every $x \in X$, where $y$ is the $z$-representer for $\varphi$.

Proof. $\quad(1) \Rightarrow(2) \Rightarrow(3)$ is obvious.

$(3) \Rightarrow(4)$ If some $x \in X \backslash[H, z]$ has a $z$-best approximation $y_{0} \in H$, then by corollary 4 ,

$$
\left\|x-y_{0}, z\right\|=\frac{1}{\|\varphi\|}|\varphi(x, z)-c|=\frac{1}{\|\varphi\|}\left|\varphi\left(x-y_{0}, z\right)\right|
$$

setting

$$
y=\frac{\left|\varphi\left(x-y_{0}, z\right)\right|}{\varphi\left(x-y_{0}, z\right)\left\|x-y_{0}, z\right\|}\left(x-y_{0}\right)
$$

we see that $\|y, z\|=1$ and $\varphi(y, z)=\|\varphi\|$. Thus $\varphi$ attains its 2-norm at $y$.

$(4) \Rightarrow(5)$ It is consequence of theorem 7 .

(5) $\Rightarrow(1)$ Let $y \in X$ be the $z$-representer of $\varphi$. Then $\varphi(x, z)=\langle x, y \mid z\rangle$, for all $x \in X$ and $\|y, z\|=\|\varphi\|$. Set

$$
w=x-\frac{(\langle x, y \mid z\rangle-c)}{\|y, z\|^{2}} y=x-\frac{\varphi(x, z)-c}{\|\varphi\|^{2}} y .
$$

Then $\varphi(w, z)=c$, so $w \in H$, and

$$
\|x-w, z\|=\frac{|\varphi(x, z)-c|}{\|\varphi\|^{2}}\|y, z\|=\frac{|\varphi(x, z)-c|}{\|\varphi\|}=d_{z}(x, H) .
$$

Thus $w=P_{H, z}(x)$.

\section{References}

[1] M. Abrishami Moghaddam and T. Sistani, Best approximation for convex subsets of 2-inner product spaces, J. Nonlinear Anal. \& Optim., 3 (2) (2012), 269-278.

[2] Y. J. Cho, P. C. S. Lin, S. S. Kim and A. Misiak, Theory of 2-inner product spaces, Nova Science Publishes, Inc, New York (2001). 
[3] S. S. Dragomir, Best approximation in pre-Hilbertian spaces and applications to continuous sublinear functionals, Anal. Uni. Timisoara, Ser St. Math, 22 (1991), 137-140.

[4] S. Elumalai, Best approximation sets in 2-normed spaces, Commu. Korean. Math. Soc., 12 (1997), 619-629.

[5] A. Misiak, n-inner product spaces, Math. Nachr., 140 (1989), 299-319.

[6] S. S. Kim and S. S. Dragomir, Some characterizations of best approximation element from subspaces in linear 2-normed spaces, East Asian Math. J., 16 (2) (2000), 205-214.

[7] S. Gähler, Lineare 2-normierte Räume, Math. Nachr., 28 (1964) 1-43.

[8] C.R. Diminnie, S. Gähler, A. White, 2-inner product spaces I, Demonstr. Math., 6 (1973), 525-536.

[9] C.R. Diminnie, S. Gähler, A. White, 2-inner product spaces II, Demonstr. Math., 10 (1977), 169-188.

[10] S. S. Kim and M. Crasmareanu, Best approximations and orthogonalites in 2k-inner product spaces, Bull. Korean. Math. Soc, 43 (2) (2006), 377387.

[11] Sh. Rezapour, Proximinal subspaces of 2-normed spaces, Anal. Theory. Appl., 22 (2) (2006), 114-119.

[12] I. Singer, Best approximation in normed linear space by element of linear subspaces, Springer, Berlin (1970).

[13] T. Sistani and M. Abrishami Moghaddam, Some results on best approximation in convex subset of 2-normed spaces, Int. J. Math. Anal., 21 (3) (2009), 1043-1049. 